\title{
The Mass of the Compact Object in the X-Ray Binary Her X-1/HZ Her
}

\author{
M. K. Abubekerov ${ }^{1}$, E. A. Antokhina ${ }^{1}$, A. M. Cherepashchuk ${ }^{1}$, \\ V. V. Shimanskii ${ }^{2}$ \\ ${ }^{1}$ Sternberg Astronomical Institute, Russia \\ ${ }^{2}$ Kazan State University, Russia
}

We have obtained the first estimates of the masses of the components of the Her $\mathrm{X}-1 / \mathrm{HZ}$ Her X-ray binary system taking into account non-LTE effects in the formation of the $\mathrm{H}_{\gamma}$ absorption line: $m_{x}=1.8 M_{\odot}$ and $m_{v}=2.5 M_{\odot}$. These mass estimates were made in a Roche model based on the observed radial-velocity curve of the optical star, HZ Her. The masses for the X-ray pulsar and optical star obtained for an LTE model lie are $m_{x}=0.85 \pm 0.15 M_{\odot}$ and $m_{v}=1.87 \pm 0.13 M_{\odot}$. These mass estimates for the components of Her X-1/HZ Her derived from the radial-velocity curve should be considered tentative. Further mass estimates from high-precision observations of the orbital variability of the absorption profiles in a non-LTE model for the atmosphere of the optical component should be made. 


\section{INTRODUCTION}

The X-ray source Her X-1 was detected by the Uhuru satellite in 1972 [1] and identified with the variable star HZ Her [2, 3, 4, 5]. The Her X-1/HZ Her binary system consists of the A7 optical component [6], which fills its Roche lobe, and an X-ray pulsar with a period of $1.24 \mathrm{~s}$. The orbital period of the binary is Porb $=1.7 \mathrm{~d}$ [7]. The system also displays 35-day X-ray variability due to the precession of the warped accretion disk [1, 8]. The optical variability of the binary is mainly due to X-ray heating of the optical star [3, 5].

The Her X-1/HZ Her binary has been thoroughly studied: the high-precision halfamplitude of the radial-velocity curve for the X-ray pulsar $K_{x}=169.049 \mathrm{~km} / \mathrm{s}$ [9] has been derived from X-ray timing, and the orbital inclination $i=81^{\circ}-88^{\circ}$ [6, 10] from the duration of the X-ray eclipses. However, no unambiguous estimates for the mass of the $\mathrm{X}$-ray pulsar have been obtained, due to the very intense X-ray heating, $k_{x} \simeq 150$.

The value of a neutron star in the Her X-1/HZ binary is not determined unambiguously. According to Middleditch and Nelson [11], the mass of the X-ray pulsar is $m_{x} \simeq 1.30 \pm 0.14 M_{\odot}$. Koo and Kron [12] suggest that the mass of the compact object in Her X- 1 is close to $m_{x} \simeq 1.5 M_{\odot}$. The mass estimated by Hutchings et al. [13] is $m_{x}=0.93 \pm 0.07 M_{\odot}$, while Reynolds et al. [10] conclude this mass lies in the interval $m_{x}=1.5 \pm 0.3 M_{\odot}$. These estimates were made using point-mass models, except for the estimate of Koo and Kron [12], which was made for a Roche model assuming a spherical shape for the optical component and roughly taking into account heating of this component.

The difficulty in accurately estimating the pulsar mass is essentially due to the intense X-ray heating of the optical component. The X-ray luminosity of the compact object is $L_{x}=3.39 \cdot 10^{37} \mathrm{erg} / \mathrm{s}$ [6], causing the spectral type of the optical star to vary from A7 $(\phi=0.0)$ to B3-B6 $(\phi=0.5)$ over half the orbital cycle [6, 14]. The temperature distribution over the surface of the optical star is also complicated by shielding of the $\mathrm{X}$-ray radiation by the warped, precessing accretion disk. Together, these factors hinder efforts to measure a high-precision, purely orbital radial velocity curve, and thereby derive an unambiguous estimate for the mass of the X-ray pulsar.

Note that, in a point-mass model, only the halfamplitude of the radial-velocity curve, $K_{v}$, is taken into account, not its shape. However, it is known that the observed radialvelocity curves of stars in binary systems may differ from the radial-velocity curves for the centers of mass, due to tidalrotational distortion of the stellar surfaces and to heating by the radiation from their companions (see the studies of Wilson and Sofia [15], Milgrom [16], and references therein). It is particularly important to take these effects into account when determining themasses of relativistic objects in X-ray binaries. For example,Milgrom [17, 16] showed that, even in the case of relatively low X-ray heating of the optical star (as in Cyg X-1 and SMC X-1), the radial velocities derived from absorption lines can vary substantially. Using profile calculations for various absorption lines in the spectra of HZ Her and other stars [17, 16, he noted the important role of the "soft" X-ray radiation $(E<1 \mathrm{keV})$ in the spectrum of the relativistic object, which is responsible for the emission components of the lines. Milgrom and Salpeter [18] developed a procedure for calculating the radiation emerging from the atmosphere of a star irradiated by X-ray radiation, intended for the calculation of line profiles. Unfortunately, in the 1970s, such 
calculations required enormous amounts of computer time, and could not be applied to the massive calculations required to solve this problem.

Antokhina et al. [30, 31] suggested a procedure for calculating line profiles and radialvelocity curves of optical stars in X-ray binaries taking into account the incident Xray flux from the relativistic object. Using this technique, we carried out a series of studies analyzing spectroscopic observations of close X-ray binaries [19, 20, 21, 27], and demonstrated the need to take into account the shape of the radial-velocity curve when determining the component masses, particularly in the presence of intense X-ray heating.

Here, we analyze the observational data of [10, in a Roche model exactly taking into account the effect of X-ray heating of the optical star [28, 29, 31], with the aim of reliably estimating the mass of the compact object in Her X-1/HZ Her. Running ahead for a moment, we note that no emission components are visible in the absorption lines in the spectrum of the optical star HZ Her, despite the extremely intense X-ray heating. Since this may be related to deviations from LTE, we have analyzed the radial-velocity curve using both LTE and non-LTE models.

\section{OBSERVATIONS}

Let us briefly describe the spectral data adopted from [10]. The observations were carried out on June 10-16, 1995 with the 2.5-m Isaac Newton Telescope of the Royal Greenwich Observatory at La Palma (Canary Islands). In total, 59 spectra at wavelengths of 4080-4940 $\AA$ and with exposures from 1200-1800 secunds were obtained with the Intermediate Dispersion Spectrograph equipped with a 235-mm camera, a diffraction grating with a dispersion of 1200 lines/mm, and a 1024x1024 pix Tektronix CCD array. The inverse linear dispersion of the spectrograms was $0.84 \AA /$ pixel. The signal-to-noise ratios in the spectra obtained were $S / N=50-100$ The wavelength scale was calibrated with a $\mathrm{Cu}-\mathrm{Ar}$ standard; the calibration error was $<0.05 \AA$.

The radial velocity of HZ Her was determined via a cross-correlation analysis using the radial velocities of IAU standard F stars observed on the same nights as HZ Her. The epoch of the maximum positive radial velocity of the optical component $(\mathrm{JD}=2448800.537)$ was used as the zero phase in [10]. We adopted the orbital period $P_{\text {orb }}=1^{d} .700167412[7]$.

Here, we took the zero orbital phase to correspond to the middle point of the eclipse of the X-ray source by the optical component. The data analysis of [10] shows that, in this case, the maximum positive radial velocity of the optical star falls at phase $\phi=0.22$, and the systematic radial velocity of the center of mass is $\gamma=-69 \mathrm{~km} / \mathrm{s}$. Figure 1 presents the observed radial-velocity curve.

The spectrograms of [10, were obtained for the orbital-phase interval of the 35-day precessional cycle of the accretion disk $\phi_{35}=0.14-0.33$ (the zero phase was ascribed to the moment when the X-ray source "switches on" for an observer on Earth). Thus, at the epoch of the spectroscopical observations [10], the accretion disk did not shield the

optical star from the X-ray flux, and our fitting of the observed radial-velocity curve did not take into account this shielding. 


\section{MODEL OF THE BINARY AND PROCEDURE FOR CALCULATING THE RADIAL-VELOCITY CURVE}

We synthesized theoretical absorption-line profiles and radial-velocity curves for the optical star using the two procedures described in detail in [28, 29, 31, which we call Procedure I [28, 29] and Procedure II [30, 31]. These procedures are similar, and differ only in the means used to calculate the radiation flux from local areas of the star. Procedure I (the more rapid algorithm) uses the Balmer line profiles tabulated by Kurucz [32]; in Procedure II (the more accurate algorithm), the emergent flux is calculated using a model atmosphere in the presence of incident external flux. We will briefly describe the calculation technique.

In a close binary, the optical component has a tidally distorted shape and an inhomogeneous temperature distribution over its surface, due to the effects of gravitational darkening and surface heating by the X-ray radiation from the relativistic object. Therefore, to model the radial-velocity curve, the X-ray binary was represented by the optical star in the Roche model and an X-ray point source with a finite mass. The tidally deformed stellar surface was divided into $\sim 2600$ elements, for each of which the emergent radiation was calculated. The calculations of the flux from a surface element included gravitational darkening, the heating of the stellar surface by radiation from its companion (the reflection effect), and limb darkening. In Procedure I [28, 29], the heating of the star's atmosphere by X-ray radiation from the companion was calculated by adding the emergent and incident flux without considering radiative transfer in the stellar atmosphere.

For each visible surface element with temperature $T_{l o c}$ and local gravitational acceleration $g_{l o c}$, the absorption-line profiles and their equivalent widths were interpolated from the Kurucz tables for Balmer lines [32]. Summing the local profiles over the visible surface of the star, taking into account the Doppler effect, and normalizing the profiles to the continuum level for each surface element, we calculated the integrated profile from the star at a given orbital phase (see [28, 29] for details). The calculated integrated absorption-line profile was used to determine the radial velocity of the star. The radial velocity at a given orbital phase was calculated for the average wavelength at the levels of the residual intensities at $1 / 3,1 / 2$, and $2 / 3$ of the integrated absorption profile.

In the more modern Procedure II, for each surface element, the parameter $k_{x}^{\text {loc }}$, equal to the ratio of the incident X-ray flux and emergent radiation flux, is calculated separately from the local temperature $T_{l o c}$ and local gravitational acceleration $g_{l o c}$, without allowing for the external irradiation of the atmosphere. The model atmosphere is calculated for these parameter values at a given point of the surface, assuming LTE, by solving the equations of radiative transfer in the line in the presence of incident external X-ray radiation (see [30, 31] for details). Thus, the intensity of the emergent radiation in the line and continuum is calculated for each local surface element. Further, following a procedure identical to Procedure I, the integrated absorption-line profile at the given orbital phase is calculated, which is then used to determine the radial velocity of the optical star.

In summary, the main difference between Procedure II [30, 31] and Procedure I [28, 29] is that, in the former the local profile of a surface element is found by constructing an LTE model of the atmosphere and calculating the intensities of emergent radiation in the line and in continuum (taking into account reprocessing of the external X-ray radiation). In 
contrast, Procedure I uses calculated and tabulated Kurucz Balmer absorption-line profiles for various effective temperatures $T_{\text {eff }}$ and gravitational accelerations $g$. Note that, in addition to the simplified treatment of the reflection effect, this method for calculating the hydrogen absorption profiles is not entirely correct, since the theoretical line profiles in Kurucz's tables [32] are given in relative fluxes rather than intensities. However, since we use the theoretical line profiles to determine radial velocities and not for comparison with the observed profiles, we consider this approximation to be reasonable [21.

Substantial deviations from LTE can occur in the atmospheres of hot stars, particularly in the presence of external irradiation. Therefore, we modeled the Balmer line profiles with Procedure II both assuming LTE and taking into account non-LTE effects in the hydrogen atom. The equilibrium non-LTE populations of the HI levels were calculated using a technique similar to that developed by Ivanova et al. [22]. In the calculations, a 23-level model of the $H I$ atom was applied, taking into account all allowed collisional and radiative mechanisms for the atom's redistribution over the states, including those generated by the external radiation. In total, the HI model includes 153 allowed boundbound and 22 bound-free radiative transitions, 55 of which were linearized. The non-LTE populations of the levels were obtained for stellar-atmosphere models for the local surface elements using the NONLTE3 [23] package, which implements the total linearization technique of Auer and Heasley [24], modified by Ivanova et al. [22] to take into account the external radiation. The calculations included all continuum sources of opacity at optical and X-ray wavelengths and about 570000 lines from the lists of Kurucz [25]. The subsequent modeling of HI line profiles was carried out following the above standard technique, but using the non-LTE populations.

When the local profiles in Fig 2 obtained for the LTE and non-LTE models are compared, it is obvious that the $\mathrm{H}_{\gamma}$ line does not display an emission component in the non-LTE case. This is due to the strong (by a factor of four to six) overpopulation of the second level $(n=2)$ of the hydrogen atom (relative to the above levels) in the chromosphere. The source function $S_{\nu} \simeq \frac{b_{j}}{b_{i}} B_{\nu}\left(T_{e}\right)$ is proportional to the ratio of the populations of the lower and upper levels of the transition. As a result, in spite of the substantial increase of $B_{\nu}\left(T_{e}\right)$ in the hot chromosphere, $S_{\nu}$ exceeds the source function in the region of formation of the continuum; i.e., the line profile appears in absorption.

As follows from an analysis of physical processes in the $H I$ atom, an overpopulation of its low-excitated states should be characteristic of hot objects with Xray irradiation. According to [26], hydrogen is responsible for less than $20 \%$ of the total absorption at X-ray wavelengths for $T_{e}=7000 K$, and for a factor of $10^{3}$ less for $T_{e}=20000 K$.

At the same time, since the temperature in the chromosphere exceeds that in the region of continuum formation, the source function in the chromosphere dominates over the mean intensity of the radiation at all optical frequencies, $J_{\nu} \ll S_{\nu}$, where $J_{\nu}$ is the mean intensity of the radiation and $S_{\nu}$ the total source function, both at a given frequency at a given depth. Thus, radiative recombination to all $H I$ levels and subsequent spontaneous transitions, which are proportional to $S_{\nu}$, dominate over ionization processes due to both the external radiation and intrinsic radiation of the atmosphere. As a result, all $H I$ levels turn out to be overpopulated relative to the LTE approximation, with the overpopulation increasing for lower states.

These non-LTE effects should result in a strengthening of the absorption and weak- 
ening of the emission components of hydrogen lines in the spectrum of an atmosphere with substantial X-ray irradiation. We wish to emphasize that, in the spectrum of the optical star HZ Her, in spite of the huge amount of X-ray heating $\left(k_{x} \simeq 150\right)$, no emission components are observed in the profiles of absorption lines, possibly due to deviations from LTE.

\section{ANALYSIS OF THE OBSERVED RADIAL-VELOCITY CURVE}

We fitted the observed radial-velocity curve of the optical star within the Roche model. Table 1 presents the model parameters for the X-ray binary Her X-1/HZ Her.

Table 1: Parameters used in the modeling of the radial-velocity curves of the optical component in the Roche model.

\begin{tabular}{|c|c|c|}
\hline$P()$. & 1.700167412 & Orbital period \\
\hline$e$ & 0.0 & Eccentricity \\
\hline$i\left(^{\circ}\right)$ & $80 ; 88$ & Orbital inclination \\
\hline$\mu$ & 1.0 & Degree of Roche lobe filling by the optical component \\
\hline$f$ & 1.0 & $\begin{array}{l}\text { Asynchronicity factor for the rotation of the optical compo- } \\
\text { nent }\end{array}$ \\
\hline$T_{\text {eff }}(\mathrm{K})$ & 8100 & $\begin{array}{l}\text { Effective temperature of the non-perturbed optical compo- } \\
\text { nent }\end{array}$ \\
\hline$\beta$ & 0.08 & Gravitational-darkening coefficient \\
\hline$k_{x}$ & 150 & $\begin{array}{l}\text { Ratio of the X-ray luminosity of the relativistic component } \\
\text { and the bolometric luminosity of the optical component, } \\
L_{x} / L_{v}\end{array}$ \\
\hline$A$ & 1.0 & $\begin{array}{l}\text { Coefficient for reprocessing of the X-ray radiation (Procedure } \\
\text { I) }\end{array}$ \\
\hline$u$ & 0.3 & Limb-darkening coefficient \\
\hline
\end{tabular}

As is noted above, Her X-1/HZ Her displays strong X-ray heating of the atmosphere of the optical star. The X-ray luminosity of the compact object is $L_{x} \simeq 3.39 \cdot 10^{37} \mathrm{erg} / \mathrm{s}$ [6], whereas the luminosity of the optical star is $L_{v} \simeq 2.28 \cdot 10^{35} \mathrm{erg} / \mathrm{s}$ (the optical luminosity was obtained in the Roche model assuming the mean effective temperature $T_{\text {eff }}=8100 \mathrm{~K}$ [6]).

In our modeling of the heating of the atmosphere of the optical component, we used the X-ray spectrum of Her X-1 obtained with BeppoSAX in 1998 [33]. In spite of the strong interstellar absorption, soft X-ray radiation from Her X-1 at $0.1-1 \mathrm{keV}$ is reliably detected, which is essential to study the conditions for the formation of the optical spectrum of HZ Her [17]. For example, Fig 3 presents two model LTE spectra of the $\mathrm{H}_{\gamma}$ hydrogen absorption line of the optical component calculated with and without taking into account the soft component (0.1-1 keV) of the irradiating X-ray spectrum. When this soft component is taken into account, the emission component in the $\mathrm{H}_{\gamma}$ absorption line is appreciably stronger.

The X-ray spectrum at 0.1-10 keV, corrected for the interstellar absorption, was reconstructed from the data of [33] using the XSPEC code. The two-component model of 
the spectrum was taken from [33]: blackbody radiation with the temperature $0.093 \mathrm{keV}$ (which dominates up to $\sim 1 \mathrm{keV}$ ), and power-law-like radiation with spectral index 0.74 (which dominates at energies exceeding $1 \mathrm{keV}$ ). We did not include the components from [33] that describe the X-ray iron emission lines due to their small contribution to the total X-ray flux.Whenmodeling the optical spectrum of HZ Her, we assumed the X-ray radiation of the relativistic object to be isotropic.

Since the exact mass of the optical star in the Her X-1/HZ Her system is not known, we adopted the masses of both components $m_{x}, m_{v}$ as parameters to be fit. We obtained the fit via an exhaustive search over the parameters, making it possible to study the surface of residuals in terms of various parameters in detail.

The half-amplitude of the radial-velocity curve of the X-ray pulsar, $K_{x}=169.049$ $\mathrm{km} / \mathrm{s}$ [9], is known from X-ray timing, and consequently the mass function of the pulsar is $f_{x}(m)=0.85 M_{\odot}$. Therefore, in the course of the exhaustive search over the component masses, we particularly made sure that the mass function of the X-ray pulsar retained the value $f_{x}(m)=0.85 M_{\odot}$. This was done as follows.

The mass function

$$
f_{x}(m)=\frac{m_{v}^{3} \sin ^{3} i}{\left(m_{x}+m_{v}\right)^{2}} .
$$

for a given orbital inclination $i$ specifies an unambiguous relation between the masses of the optical star $m_{v}$ and the X-ray pulsar $m_{x}$.

A value of $m_{x}$ was calculated from (11) for each value of $m_{v}$ and a fixed value of $i$. Further, the theoretical radial-velocity curve for the optical component was determined based on the resulting component masses $m_{x}$ and $m_{v}$, with the numerical parameters of the Roche model taken from Table 1. The adequacy of the model to the observational data was tested using the statistical $\chi^{2}$ criterion, selecting a significance level of $5 \%$ (see 34] for details).

\section{Results Obtained with Procedure I}

Since the radial velocities of [10] were derived from spectra obtained with the response function $\mathrm{FWHM}=1.7 \AA$, we calculated the theoretical radial-velocity curves from the pro-

file of the $\mathrm{H}_{\gamma}$ line convolved with an instrumental profile with FWHM=1.7 $\mathrm{A}$. Figure 4 presents the theoretical integrated profiles of the $\mathrm{H}_{\gamma}$ absorption line.

Table 2: Mass of the relativistic and optical components of Her X-1 obtained in the Roche model with Procedure I.

\begin{tabular}{|c|c|c|}
\hline $\begin{array}{c}\text { Orbital } \\
\text { inclination }\end{array}$ & $m_{x}\left(M_{\odot}\right)$ & $m_{v}\left(M_{\odot}\right)$ \\
\hline$i=80^{\circ}$ & 1.84 & 2.6 \\
$i=88^{\circ}$ & 1.78 & 2.5 \\
\hline
\end{tabular}

Table 2 presents the masses of the X-ray pulsar $m_{x}$ and optical star $m_{v}$ corresponding to the minimum $\chi^{2}$ residual for the theoretical and observed radial-velocity curves. In 
Table 2, the component masses are given without errors, since models of the binary were rejected according to the selected significance level $\alpha=5 \%$. For the quantile $\Delta(\alpha=5 \%)=$ 77.93 , the minimum residual for the orbital inclinations $i=80^{\circ}, i=88^{\circ}$ was $\chi_{\min }^{2} \simeq 170$. Figure 5 presents the theoretical radial-velocity curve and Fig 6 a the behavior of the $\chi^{2}$ residual for $i=88^{\circ}$.

\section{Results Obtained with Procedure II}

It was noted above, we used the observational data of [10]. In those spectra, the profile of the $\mathrm{H}_{\gamma}$ absorption line at orbital phase 0.47 displays a distortion [[10, Fig. 1]. At the same orbital phase, the theoretical integrated profile of the $\mathrm{H}_{\gamma}$ absorption convolved with a response function with $\mathrm{FWHM}=1.7 \AA$ observed profile (Fig. 7). We were able to reproduce a similar distortion in the integrated theoretical profile of the $\mathrm{H}_{\gamma}$ hydrogen line at phase 0.47 only with a response-function width of $\mathrm{FWHM}=5 \AA$ (Fig. 7). Therefore, we carried out our analysis of the observed radial-velocity curve for the $\mathrm{H}_{\gamma}$ hydrogen line with the response-function width $\mathrm{FWHM}=5 \AA$.

For the effective temperature of the stellar surface $\sim 8000 \mathrm{~K}$, the hydrogen line displays a full width at half maximum of the residual intensity of several tens of $\AA$. In our case, the width of the model integrated profile of the $\mathrm{H}_{\gamma}$ line at this level was $\sim 25 \AA$ (Fig. 8). Therefore, we faced the problem of determining the radial velocity from such a broad $\mathrm{H}_{\gamma}$ absorption profile. For Procedure I, the half-width of the model profile was smaller $\sim 10 \AA$ (Fig. 8), and the profile itself was more symmetrical. Therefore, to determine the position of the center of gravity of the line, it was sufficient to take three cross sections at heights of $1 / 3,1 / 2$, and $2 / 3$ of the residual intensity. If the integrated $\mathrm{H}_{\gamma}$ profile is calculated with Procedure II, three cross sections are not sufficient, and we selected six and eight levels of the cross section of the residual intensity of the model integrated profile to determine the center of gravity of the $\mathrm{H}_{\gamma}$ line.

Table 3: Masses of the relativistic and optical components obtained from the radial velocity determined from six cross sections of the integrated $\mathrm{H}_{\gamma}$.

\begin{tabular}{|c|c|c|}
\hline $\begin{array}{c}\text { Orbital } \\
\text { inclination }\end{array}$ & $m_{x}\left(M_{\odot}\right)$ & $m_{v}\left(M_{\odot}\right)$ \\
\hline$i=80^{\circ}$ & $0.85 \pm 0.15$ & $1.87 \pm 0.13$ \\
$i=88^{\circ}$ & $0.81 \pm 0.13$ & $1.80 \pm 0.11$ \\
\hline
\end{tabular}

In the former case, the radial velocity was determined from the wavelength averaged over cross sections at six levels of the lower half of the absorption profile; the resulting error in the radial velocities were $\sim 1 \mathrm{~km} / \mathrm{s}$. When eight cross-section levels were used, two cross sections in the upper half of the model profile of the $\mathrm{H}$ absorption line were introduced in addition to the six for the previous case; the resulting error in the theoretical radial velocities were $\sim 2.5 \mathrm{~km} / \mathrm{s}$. Here, we take the radial velocity at orbital phase $\phi=0.0$ (when the optical component eclipses the X-ray source) as the error in the theoretical radial velocity. The error in the radial velocity for the calculation using six cross sections 
is appreciably smaller due to the narrowness of the lower part of the $\mathrm{H}_{\gamma}$ profile compared to the wings (Fig. 8).

The mass of the X-ray pulsar $m_{x}$ and optical star $m_{v}$ corresponding to the minimum $\chi^{2}$ residual for the theoretical and observed radial-velocity curves obtained using six and eight cross sections of the integrated $\mathrm{H}_{\gamma}$ line profile are presented in Tables 3 and 4, respectively. Figure $\mathrm{H}_{\gamma}$ presents the radial-velocity curve obtained using six cross sections of the integrated 5 profile.

It is obvious that the results depend on the method used to determine the center of gravity of the integrated $\mathrm{H}_{\gamma}$ absorption profile, although, within the errors, the mass of the X-ray pulsar is consistent with that of the optical star.

Table 4: Masses of the relativistic and optical components obtained from the radial velocity determined from eight cross sections of the integrated $\mathrm{H}_{\gamma}$ profile.

\begin{tabular}{|c|c|c|}
\hline $\begin{array}{c}\text { Orbital } \\
\text { inclination }\end{array}$ & $m_{x}\left(M_{\odot}\right)$ & $m_{v}\left(M_{\odot}\right)$ \\
\hline$i=80^{\circ}$ & 0.70 & 1.75 \\
$i=88^{\circ}$ & 0.70 & 1.70 \\
\hline
\end{tabular}

The model of the binary obtained using six cross sections is consistent with the observational data at the $\gamma=95 \%$ confidence level, with the minimum residual $\chi_{\text {min }}^{2} \simeq 70$ (Fig,6b). When eight cross sections are used, the binary model is rejected at the significance level $\alpha=5 \%$, with the minimum residual $\chi_{\text {min }}^{2} \simeq 90$ (for this reason, the component masses in Table 4 are presented without errors).

\section{Integrated Profile and the Radial-velocity Curve Including non-LTE Effects}

As is noted above, in spite of the substantial X-ray heating $\left(k_{x}=150\right)$, the absorption lines in the optical spectrum of the HZ Her system display no visible emission components. This is apparently due to non- LTE effects (see above).

We also calculated the integrated $\mathrm{H}_{\gamma}$ absorption profiles and corresponding radialvelocity curve in a non-LTE model (Fig. 5). These calculations were performed using the NONLTE3 code [23] for the model binary parameters $m_{x}=0.81 M_{\odot}, m_{v}=1.80 M_{\odot}$, and $i=88^{\circ}$. (see the remaining parameters in Table 1), taking the width of the response function to be FWHM=1.7 $\AA$. Regrettably, calculations with the NONLTE3 procedure are time consuming, which currently makes searches for the parameters of close binaries challenging, and we restrict our consideration here to qualitative conclusions.

Figures 2 and 8 present the local and integrated profiles of the $\mathrm{H}_{\gamma}$ absorption line calculated in the non-LTE approximation. Figure 5 presents the radial-velocity curve derived from these profiles.

We can see from Fig 8 that, qualitatively, the integrated $\mathrm{H}_{\gamma}$ absorption profile obtained in the non-LTE model is close to the integrated profile calculated using Procedure I - in both profiles, no emission component is seen. At the same time, the integrated 
$\mathrm{H}_{\gamma}$ absorption profile obtained assuming LTE with Procedure II displays an emission component (see Figs 3 and 8).

We can also see in Fig 5 that the shape of the radial-velocity curve obtained with the non- LTE model is qualitatively closest to that calculated with Procedure I. In this connection, the results of fitting the observed radial-velocity curve for HZ Her/Her X-1 made with Procedure I may bemore reliable: $m_{x}=1.78 M_{\odot}, m_{v}=2.5 M_{\odot}$ for the orbital inclination $i=88^{\circ}$. We stress again, however, that the model of the binary system with these parameters was rejected at the $\alpha=5 \%$ significance level adopted in the study.

\section{DISCUSSION}

Let us consider the advantages and disadvantages of the procedures used to calculate the radial-velocity curves in our study.

When the integrated profile of the optical component is calculated with Procedure I, we use integrated profiles of absorption lines of single stars tabulated by Kurucz [32 for the local profiles. These absorption profiles do not contain emission components, and the local profiles used in Procedure I are qualitatively consistent with the local profiles of the $\mathrm{H}_{\gamma}$ absorption lines in the non-LTE approximation. However, the model integrated $\mathrm{H}_{\gamma}$ profile obtained with Procedure I does not reproduce the distinctive distortion of the $\mathrm{H}_{\gamma}$ absorption line at orbital phase $\phi=0.47$ ([10], Fig. 1), and the best-fit theoretical radialvelocity curve seems to diverge from the observed radial-velocity curve both qualitatively and quantitatively $\left(\chi_{\min }^{2} \simeq 170\right.$ - the model is rejected at the $\alpha=5 \%$ significance level; Fig (5). In this light, we cannot take our estimates for the Her X-1/HZ Her component masses obtained with Procedure I to be final.

Let us consider the results of our fitting of the observational data and the specific features of calculating the model absorption profiles with Procedure II.

The local profiles assuming LTE with exact account of the effect of heating contain an emission component (which is not seen in the observed optical spectra of HZ Her), whereas the local $\mathrm{H}_{\gamma}$ absorption profiles calculated in the non-LTE approximation display no emission component (Fig (2) . Nonetheless, the theoretical integrated $\mathrm{H}_{\gamma}$ absorption profile convolved with an artificially broadened response function with FWHM $=5 \AA$ (exceeding the real width of the response function by a factor of three) qualitatively reproduce the "distortion" of the observed absorption profile shape (cf. Fig. 1 in [10] and Fig[8]). The full width of the obtained integrated $\mathrm{H}_{\gamma}$ absorption profile at half the residual intensity was $\sim 25 \AA$, hindering determination of the center of gravity of the absorption line. Six or eight cross sections were used to determine the center of gravity of the model integrated $\mathrm{H}_{\gamma}$ absorption profile.

The component masses obtained with six and eight cross sections of the model integrated $\mathrm{H}_{\gamma}$ profile are consistent within the errors (Tables 3 and 44). However, both the quantitative estimates of the mass of the binary components obtained with Procedure II and the reliability of the binary model depend on the number of cross sections of the integrated profiles used to determine the center of gravity of the $\mathrm{H}_{\gamma}$ absorption profile. For example, when eight cross sections of the model integrated $\mathrm{H}_{\gamma}$ profile were used, the model was rejected at selected significance level of $\alpha=5 \%$, and the masses for the binary components differed by $\sim 0.1 M_{\odot}$ from those obtained when six cross sections of the 
integrated profile were used.

Thus, it does not seem reasonable to give preference to any one of the various results, and we consider the results presented in Tables 2 and 3 equally valid. As we have shown here, reliable estimation of the component masses for the Her X-1/HZ Her binary system is possible only based on an analysis of the radial-velocity curve jointly with the orbital variability of the absorption profiles, with correct account for non-LTE effects.

\section{CONCLUSION}

Our study has mainly focused on the methodical side of the problem considered. We have shown that determining the radial velocities in close binaries with intense X-ray heating of the optical star requires taking into account non-LTE effects in the formation of absorption lines in the spectrum of the optical star. For Her X-1/HZ Her-type binary systems, not only the radial-velocity curve (which is specified by the line profiles only ambiguously)must be analyzed, but also line profiles at different phases of the orbital period.

Our calculations have shown that, due to the very intense X-ray heating in Her X-1/HZ Her, the $\mathrm{H}_{\gamma}$ absorption line of the optical star is appreciably asymmetrical, making it difficult to determine unambiguously the center of gravity of its profiles and, as a consequence, the radial velocities and component masses. For example, when the theoretical radial velocity is determined using different numbers of cross sections of the absorption-line profile, the mass of the X-ray pulsar $m_{x}$ varies by $\sim 15 \%$ (Tables 3 and 4). We stress that the methods for estimating the Her X-1/HZ Her component masses presented here are based on the most current and physically justified techniques, in which local absorption-line profiles are calculated taking into account external X-ray heating. Nonetheless, even this approach cannot yield unambiguous mass estimates for the X-ray pulsar in Her X-1/HZ Her, due to the enormous rate of X-ray heating in this unique X-ray binary. Analyzing the radial-velocity curve in two ways, we have obtained two masses for the X-ray pulsar: $m_{x}=0.85 \pm 0.15 M_{\odot}$ and $m_{x}=1.8 M_{\odot}$. Thus, the mass of the X-ray pulsar in Her X-1 is currently determined only to within a factor of $\sim 2$, due to uncertainty in realistic models for the formation of line profiles in the spectrum of the heated optical star.

Our results demonstrate the need to use high-resolution spectrograms $(\lambda / \Delta \lambda \simeq 50000)$ and to take into account non-LTE effects when estimating the mass of the compact object. Correct and reliable estimates for the component masses can be obtained only by analyzing also the orbital variability of the absorption-line profiles in the spectrum of the optical component in a non-LTE approximation.

We thank I.I. Antokhin for useful discussions. 


\section{References}

[1] Tananbaum H., Gursky H., Kellog E.M. // Astrophys.J. 1972 V.174, L.143.

[2] N. E. Kurochkin // Perem. Zvezdy 18, 425 (1972).

[3] Cherepashchuk A.M., Efremov Yu.N., Kurochkin N.E. et al // IBVS No.720, 1972.

[4] Forman. W., Jones C.A., Liller W. // Astrophys.J. 1972 V.177, L.103.

[5] Bahcall J.N., Bahcall N.A. // Astrophys.J. 1972 V.178 L.1.

[6] Cheng F.H., Vrtilek S.D., Raymond J.C. // Astrophys.J. 1995 V.452 P.825.

[7] Prince T.A., Bildsten L., Chakrabarty D. // in Holt S.S., Day C.S. eds, The Evolution of X-ray Binaries. AIP Press, New York, 1994 P.235.

[8] Schandl S. // Astron. Astroph. 1996 V.307, P.95.

[9] Deeter J.E., Boynton P.E., Pravdo S.H. // Astrophys.J. 1981 V.247 P.1003.

[10] Reynolds A.P., Quaintrell H., Still M.D. et al. // MNRAS 1997 V.288., P.43.

[11] Middleditch J., Nelson J.E. // Astrophys.J. 1976 V.208 P.567.

[12] Koo D.C., Kron R.C. // PASP 1977 V.89 P.285.

[13] Hutchings J.B., Gibson E.M., Crampton D., Fisher W.A. // Astrophys.J. 1985 V.292 P.670.

[14] Anderson S.F., Wachter S., Margon B. et al. // Astrophys.J. 1994 V.436 P.319.

[15] Wilson R.E., Sofia S. // Asrophys.J. 1976 V.203. P.182.

[16] Milgrom M. // Astron. Astrophys. 1977. V.54. P.725

[17] Milgrom M. // Astrophys.J. 1976. V.206. P.869

[18] Milgrom M. Salpeter E.E. // Astrophys.J. 1975. V.196. P.853

[19] M. K. Abubekerov, E. A. Antokhina, and A. M. Cherepashchuk // Astron. Rep. 48, 89 (2004).

[20] M.K. Abubekerov, E.A. Antokhina, and A.M. Cherepashchuk // Astron. Rep. 48, 550 (2004). 
[21] M. K. Abubekerov, E. A. Antokhina, and A. M. Cherepashchuk // Astron. Rep. 49, 801 (2005).

[22] D.V. Ivanova, N.A. Sakhibullin, and V.V. Shimanskii // Astron. Rep. 46, 390 (2002)

[23] N. A. Sakhibullin, Tr. Kazan. Gor. Astron. Obs. 48, 9 (1983).

[24] L.H. Auer, J. Heasley, Astrophys. J. 205, 165 (1971).

[25] R.L. Kurucz, SAO CD-Roms, (Cambridge, MA02138, USA. 1994).

[26] N. A. Sakhibullin and V. V. Shimanskii // Astron. Rep. 40, 62 (1996).

[27] M. K. Abubekerov, E. A. Antokhina, A. M. Cherepashchuk, and V. V. Shimanskii // Astron. Rep. 50, 544 (2006).

[28] E. A. Antokhina and A. M. Cherepashchuk // Astron. Rep. 38, 367 (1994).

[29] E. A. Antokhina // Astron. Rep. 40, 483 (1996).

[30] E. A. Antokhina, A. M. Cherepashchuk, and V. V. Shimanskii, Izv. Ross. Akad. Nauk, Ser. Fiz. 67 (3), 293 (2003).

[31] E. A. Antokhina, A. M. Cherepashchuk, and V. V. Shimanskii // Astron. Rep. 49, 109 (2005).

[32] Kurucz R.L. // CD-ROMs, 1993.

[33] Oosterbroek T., Parmar A.N., Martin D.D.E., Lammers U. // Astron. Astroph. 1997 V.327, P.215.

[34] A. M. Cherepashchuk // Astron. Rep. 37, 585 (1993) 


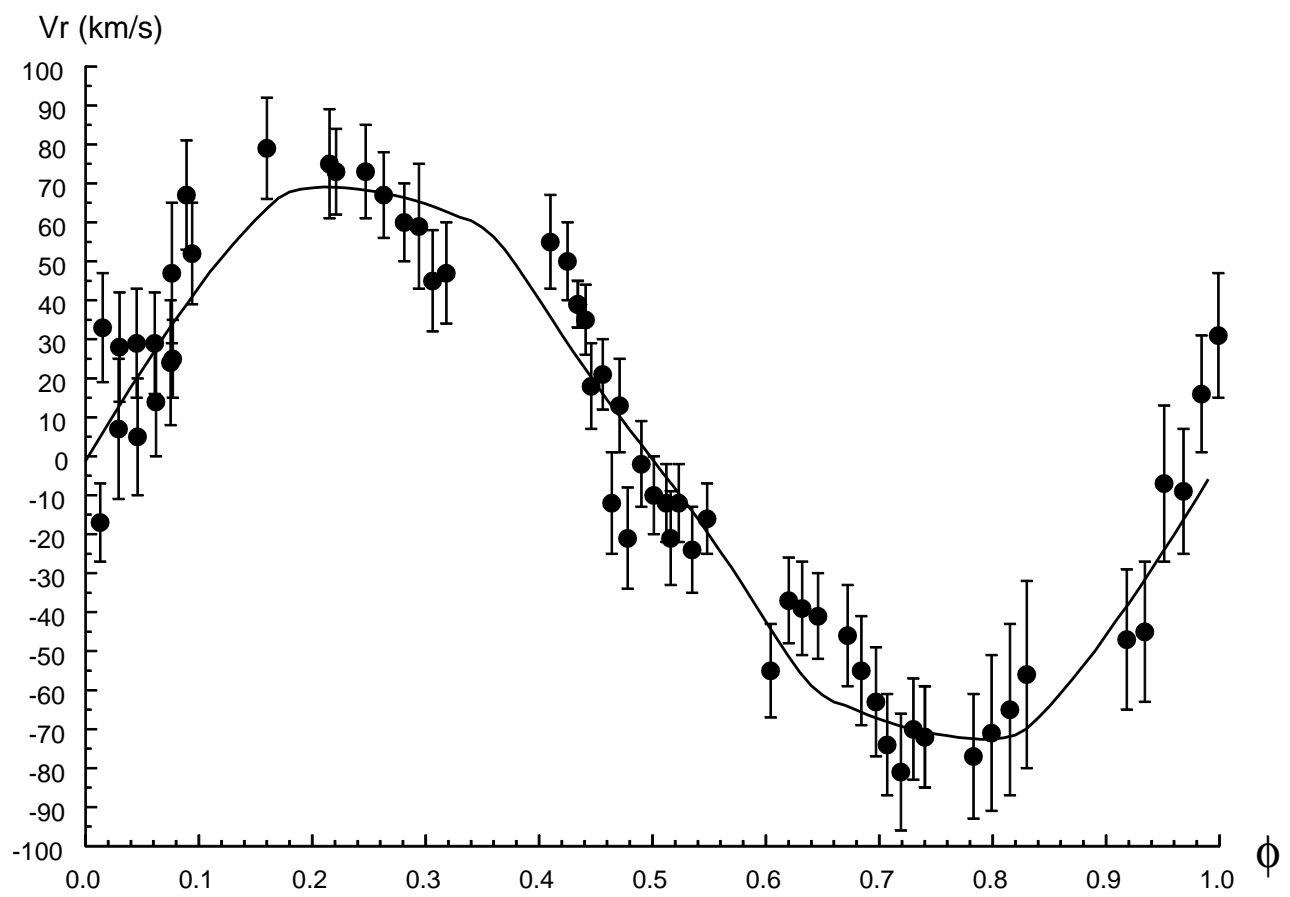

Figure 1: Observed and theoretical radial-velocity curves of the optical component of the X-ray binary Her X-1/HZ Her. The points indicate the radial velocities of HZ Her taken from [10]. The solid curve is the theoretical radial-velocity curve for a Roche model calculated using Procedure II, with the mass of the compact object $m_{x}=0.81 M_{\odot}$, the mass of the optical star $m_{v}=1.80 M_{\odot}$, and the orbital inclination $i=88^{\circ}$. (for the remaining parameters of the binary, see Table 11). 


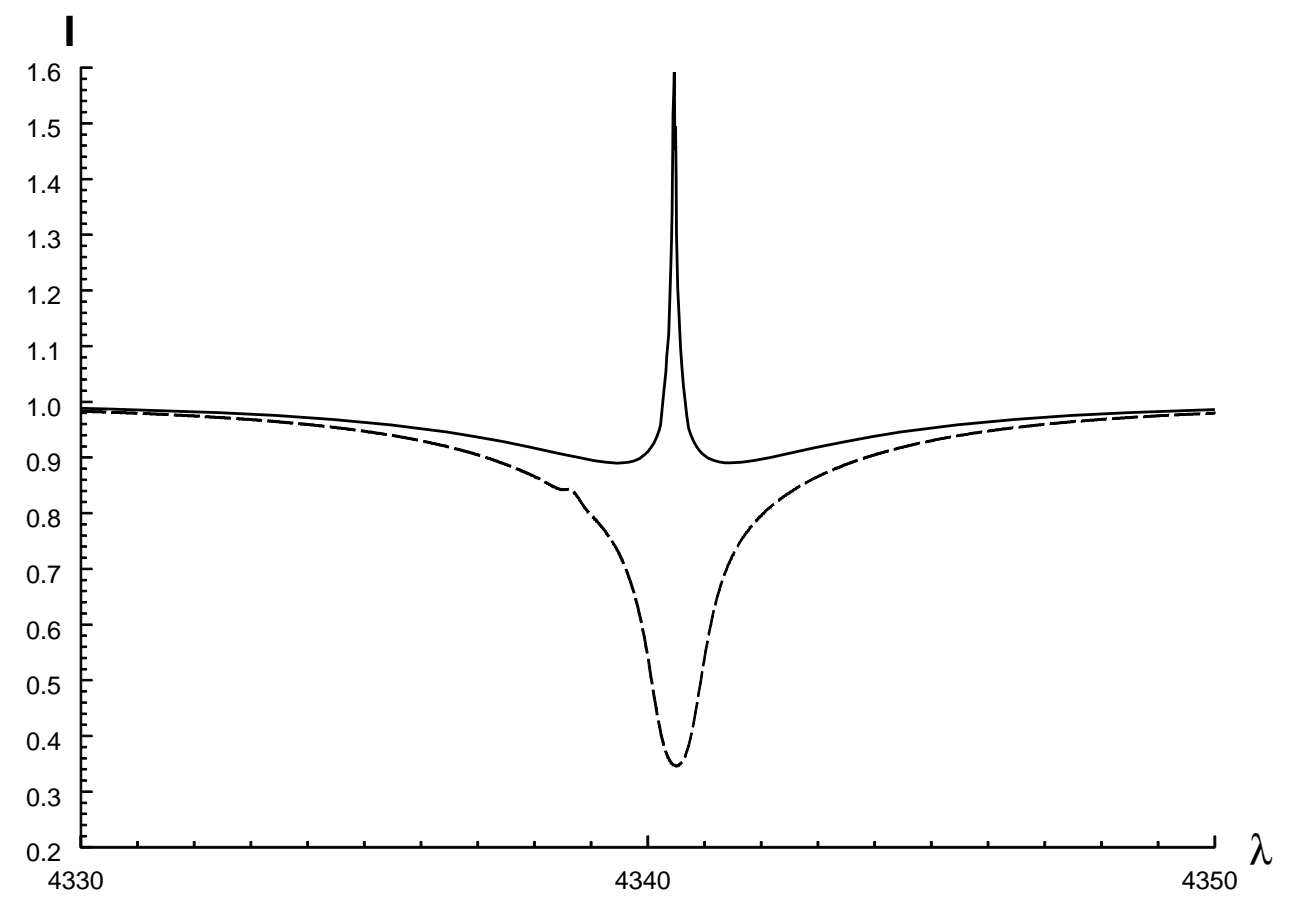

Figure 2: Model local profiles for the $\mathrm{H}_{\gamma}$ absorption line. The solid curve corresponds to the calculations assuming LTE (Procedure II) for $m_{x}=0.9 M_{\odot}, m_{v}=2.0 M_{\odot}, i=80^{\circ}$, $k_{x}=150$, and the dashed curve to the non-LTE calculations for the same parameters of the binary. The temperature and gravitational acceleration for the local surface element are $T_{l o c}=7825 \mathrm{~K}$ and $\log g_{l o c}=3.336$. 


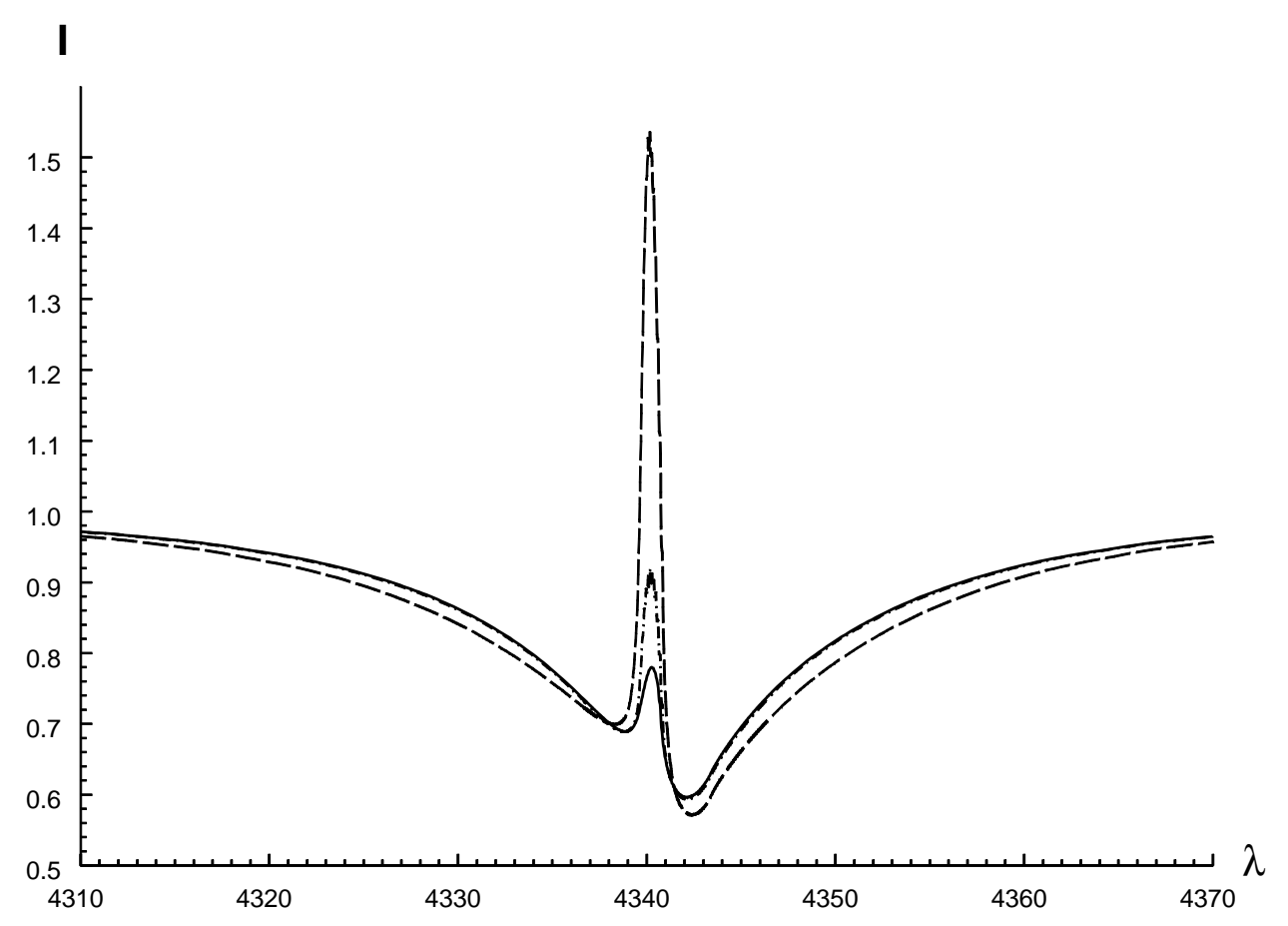

Figure 3: Effect of taking into account the soft component of the incident X-ray flux on the shape of the $\mathrm{H}_{\gamma}$ line. Integrated profiles calculated with Procedure II for $m_{x}=1 M_{\odot}$, $m_{v}=2 M_{\odot}, i=80^{\circ}, k_{x}=150$ are shown. The X-ray irradiation was modeled at 0.1-1 $\mathrm{keV}$ (dashed curve), 0.1-17 keV (dash.dotted curve), and 1-17 keV (solid curve). 


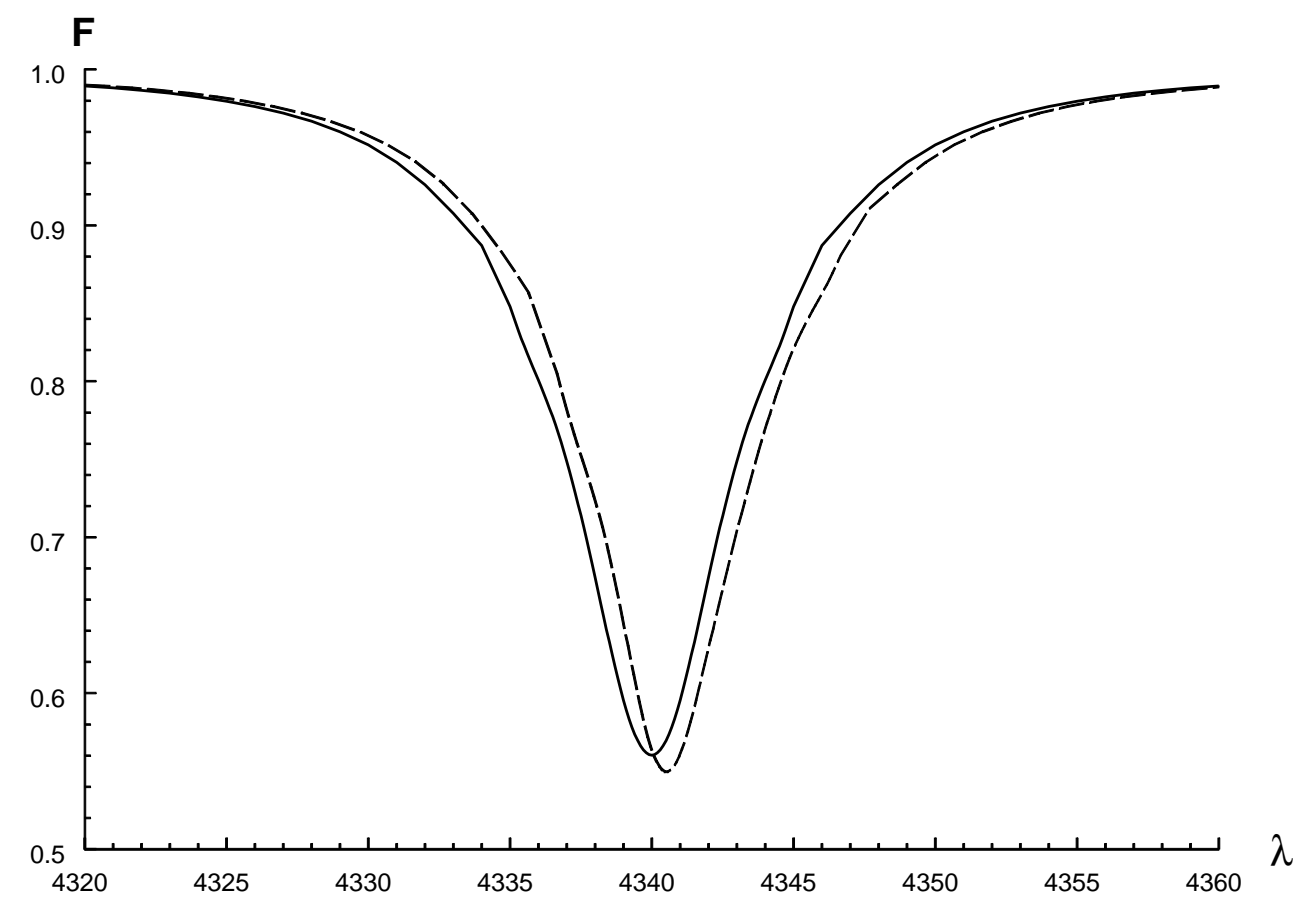

Figure 4: Integrated profiles of the $\mathrm{H}_{\gamma}$ absorption line calculated in the Roche model with Procedure I, for the width of the response function FWHM=1.7 Aand with $m_{x}=1.78 M_{\odot}$, $m_{v}=2.5 M_{\odot}$, and $i=88^{\circ}$. The solid line corresponds to the orbital phase $\phi=0.0$, and the dashed line to $\phi=0.30$. 


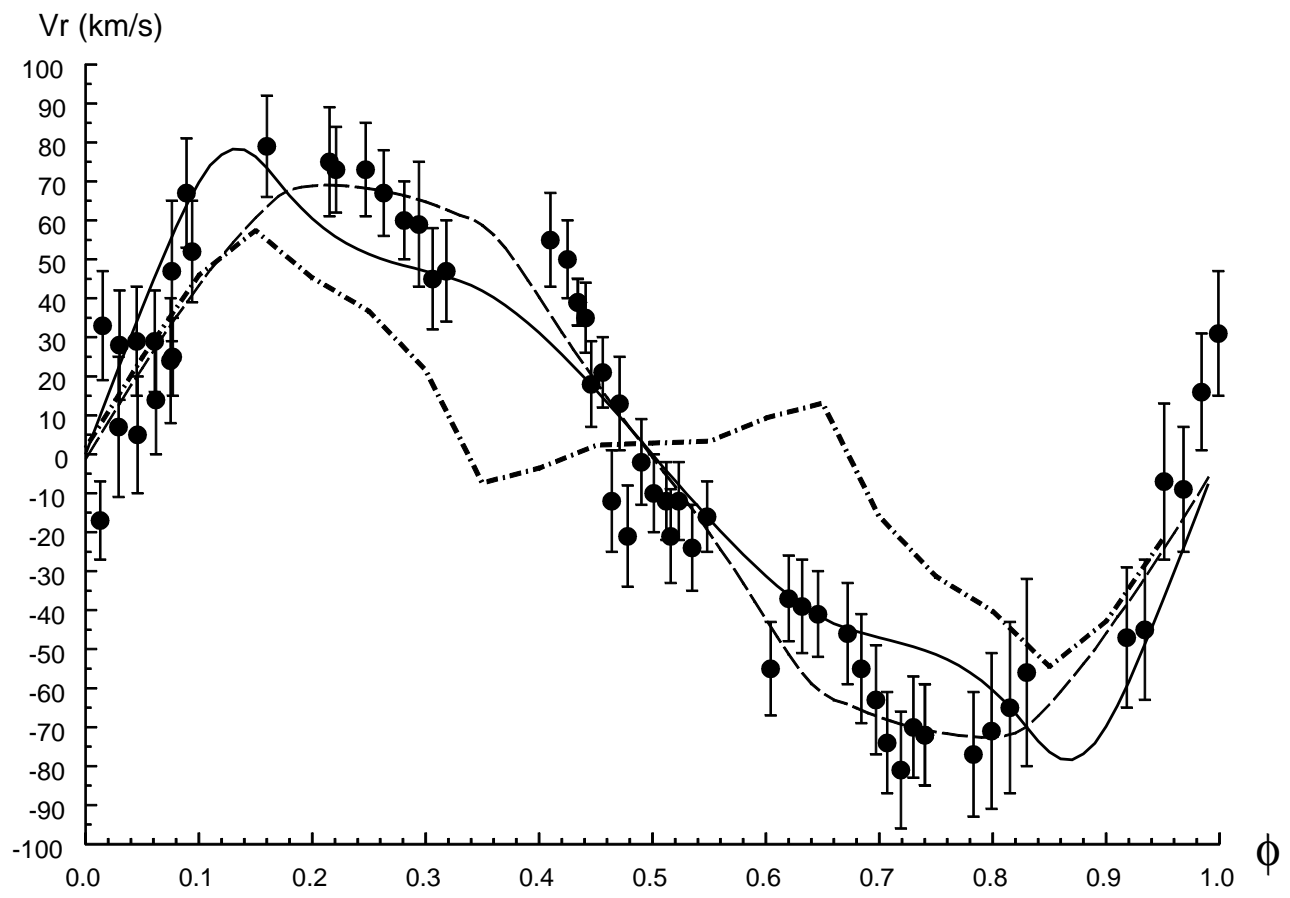

Figure 5: Observed radial-velocity curve for the optical component of the X-ray binary Her X-1 taken from [10] (points) and theoretical radial-velocity curves calculated with Procedure I for $m_{x}=1.78 M_{\odot}, m_{v}=2.5 M_{\odot}$, and $i=88^{\circ}$ (solid curve); and with Procedure II for $m_{x}=0.81 M_{\odot}, m_{v}=1.80 M_{\odot}$, and $i=88^{\circ}$ from six cross sections of the integrated profile of the $\mathrm{H}_{\gamma}$ line (dashed curve) and using the NONLTE3 [23] package in the nonLTE model for $m_{x}=0.81 M_{\odot}, m_{v}=1.80 M_{\odot}, i=88^{\circ}$. (dash-dotted curve). 


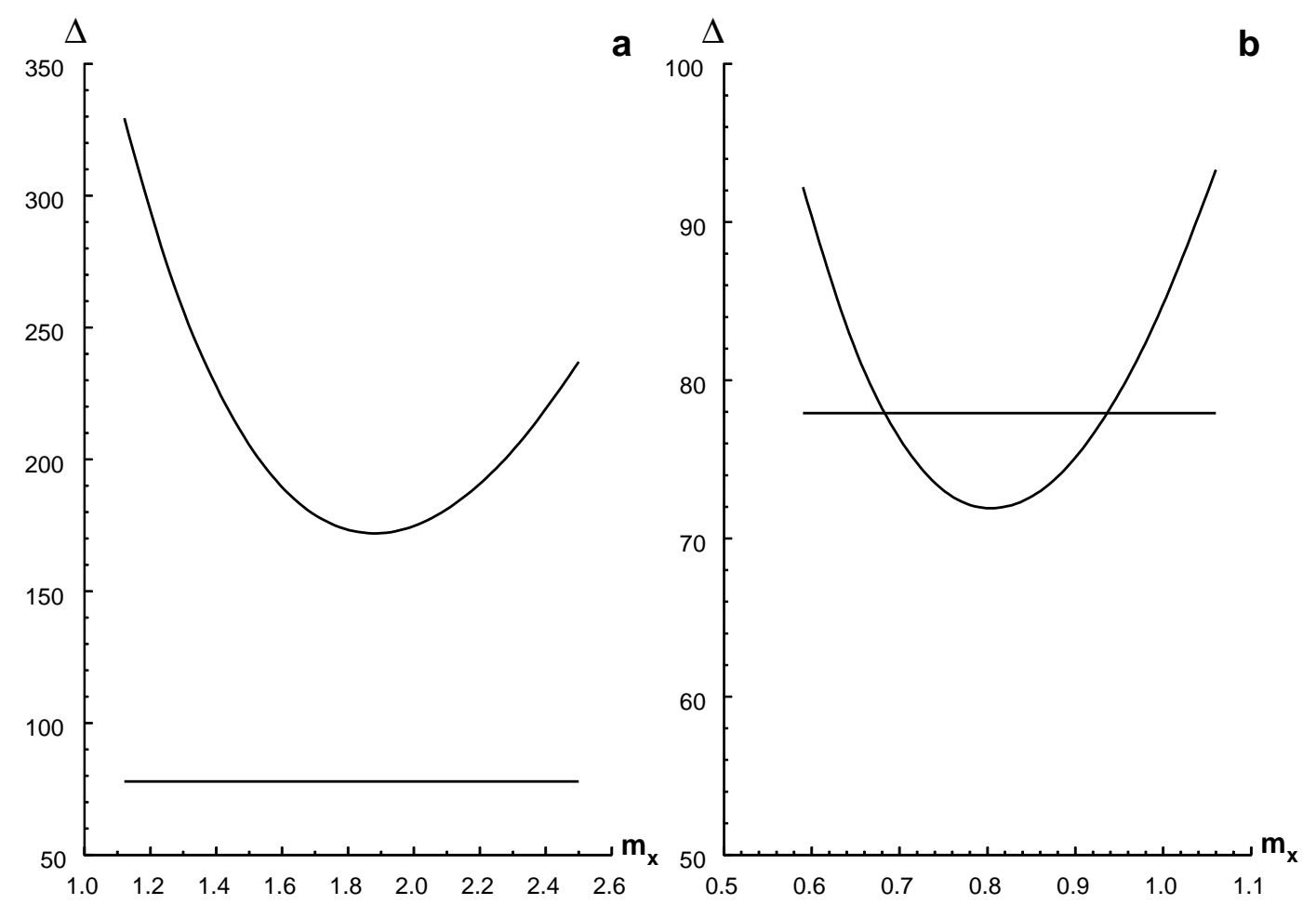

Figure 6: Discrepancies between the observed and theoretical radial velocity curves of HZ Her. The theoretical curves were obtained with (a) Procedure I and (b) Procedure II, for the orbital inclination $i=80^{\circ}$. The horizontal line corresponds to the critical level of the $\chi^{2}$ criterion $\Delta_{59}=77.93$ for the significance level $\alpha=5 \%$. 


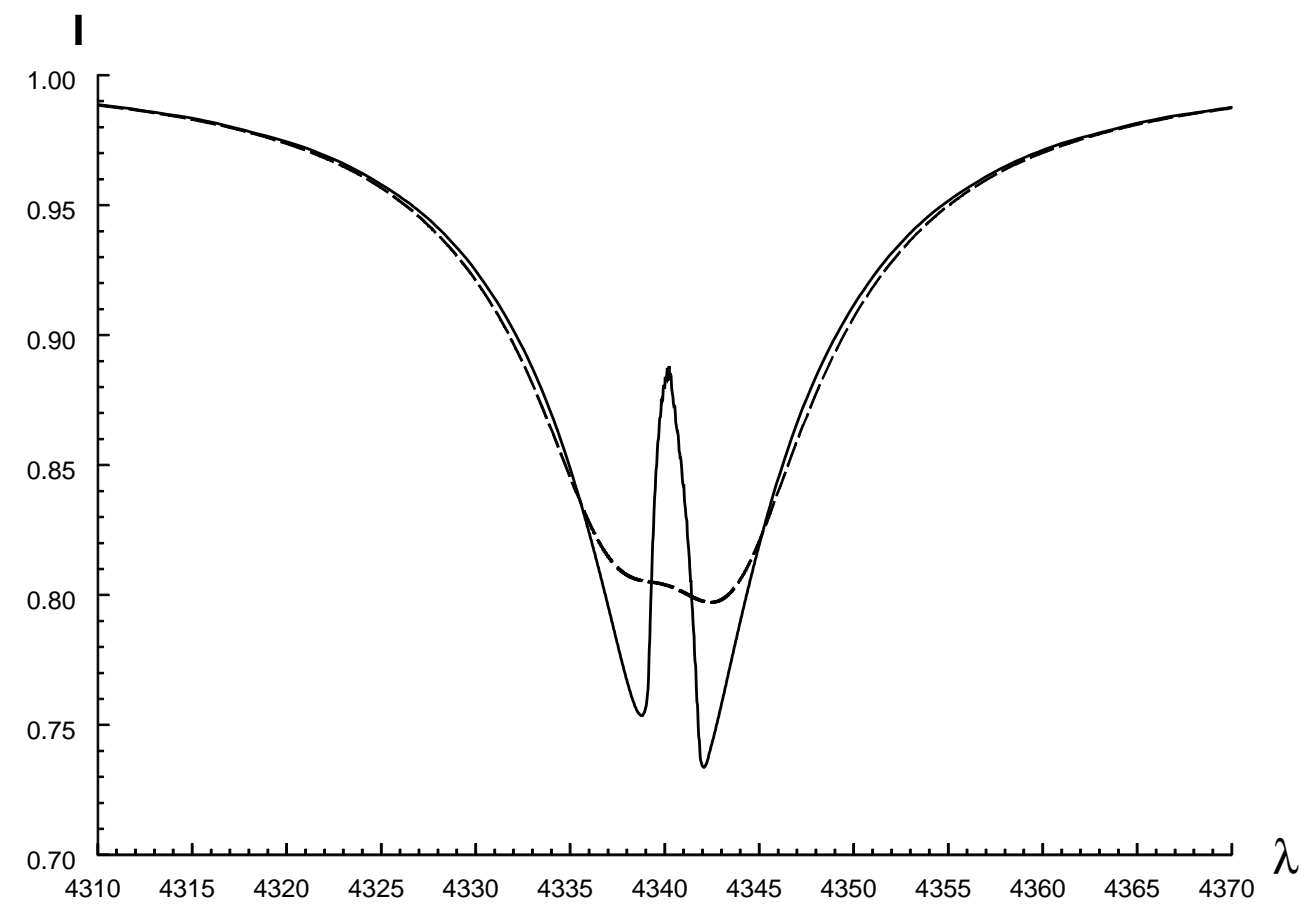

Figure 7: Model integrated profile of the hydrogen absorption line at orbital phase $\phi=0.47$, calculated assuming LTE with Procedure II for the response-function widths $\mathrm{FWHM}=1.7 \AA$, (solid curve) and $\mathrm{FWHM}=5 \AA$, (dashed curve) for $m_{x}=0.81 M_{\odot}, m_{v}=$ $1.80 M_{\odot}$, and $i=88^{\circ}$. 


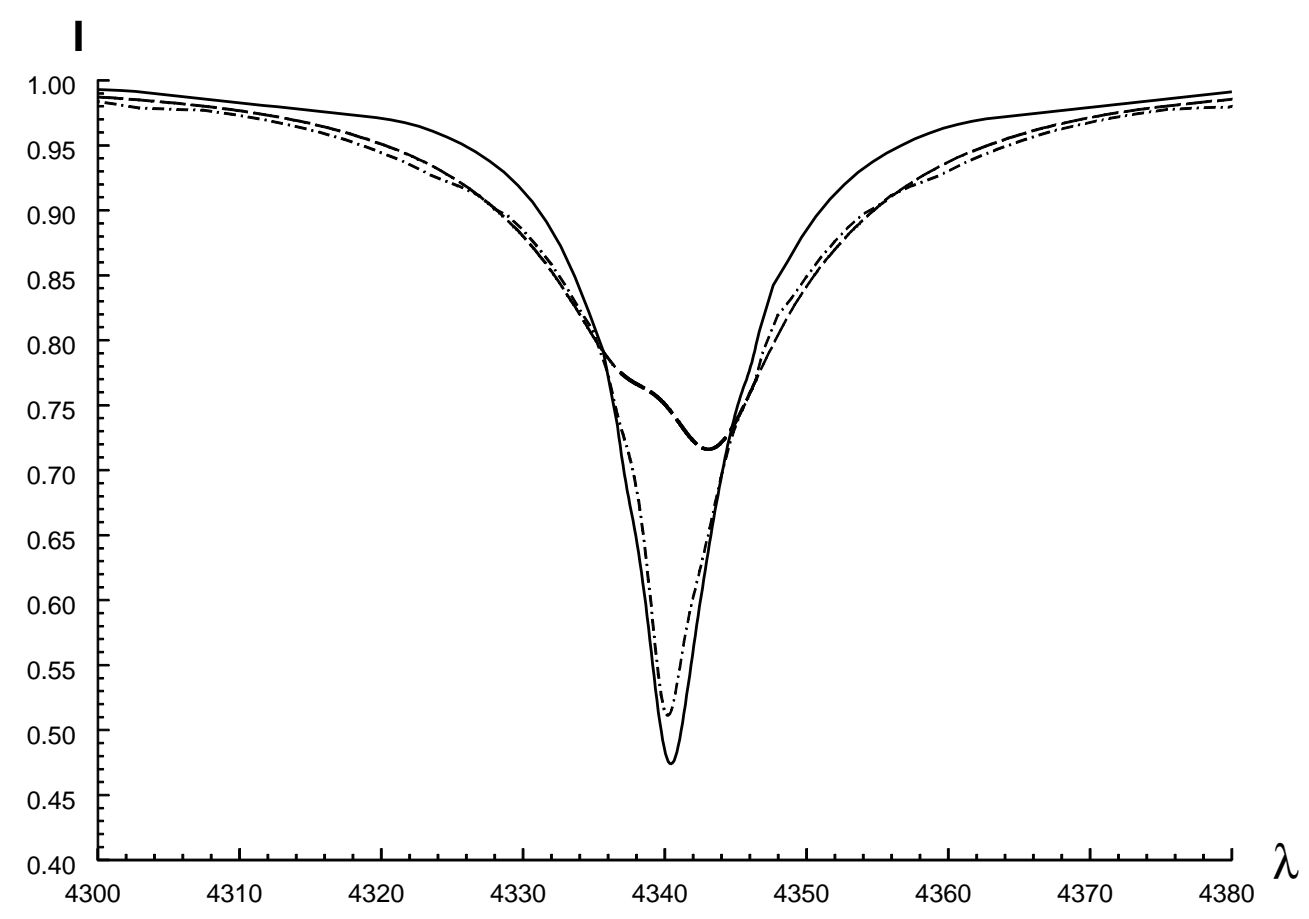

Figure 8: Model integrated profile of the $\mathrm{H}_{\gamma}$ hydrogen absorption line at orbital phase $\phi=0.30$, obtained with Procedure II for the response-function width FWHM=5 $\AA$, and $m_{x}=0.81 M_{\odot}, m_{v}=1.80 M_{\odot}$, and $i=88^{\circ}$ (dashed curve); with Procedure I for the response-function width FWHM $=1.7 \AA$, and $m_{x}=1.78 M_{\odot}, m_{v}=2.5 M_{\odot}$, and $i=88^{\circ}$ (solid curve); and with the NONLTE3 package in the non-LTE model for the responsefunction width $\mathrm{FWHM}=1.7 \AA$, and $m_{x}=0.81 M_{\odot}, m_{v}=1.80 M_{\odot}$, and $i=88^{\circ}$ (dashdotted curve). 